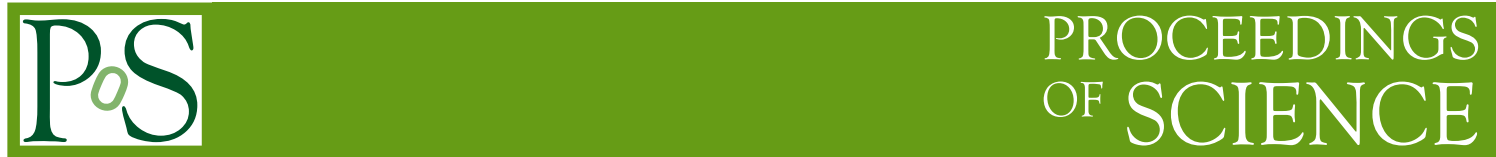

\title{
Ward-Takahashi Relations: Longitudinal and Transverse
}

Faqir Khanna*

Theoretical Physics Institute

University of Alberta, Edmonton, Alberta, T6G 2G7 Canada

E:mailkhanna@phys.ualberta.ca

\begin{abstract}
Ward-Takahashi relations in Abelian gauge theories are a consequence of the gauge symmetry that give current conservation. These are exact relations that must be satisfied in any perturbative calculation. However these relations constrain only the longitudinal part of the vertex. The transverse part of the vertex remains free of all restrictions. However Takahashi suggested a method to provide limitations on this component of the vertex. The idea has been revised and a full set of equations are established. These are checked at one-loop level. The generalised Ward-Takahashi relations provide a constraint on the complete vertex function.
\end{abstract}

Fifth International Conference on Mathematical Methods in Physics - IC2006

April 24-28 2006

Centro Brasilerio de Pesquisas Fisicas, Rio de Janeiro, Brazil

\footnotetext{
${ }^{*}$ Speaker.
} 
Ward-Takahashi relations [1] provide exact relations between Green's functions or amplitudes. These relations are based on the fundamental symmetry of the system and thus play an important role in providing a consistent description in any perturbative approach. Some non-perturbative results can be deduced from these relations. For example the appearance of Nambu-Goldstone bosons [2] as a consequence of spontaneous symmetry breaking and a consistent theory of the spin and isospin excitations in an infinite nuclear system [3] can be formulated using the WardTakahashi relations (W-T). These relations are useful in finding possible solutions of SchwingerDyson equation [4].

The Schwinger-Dyson equations contain the full structure of the quantum field theory [4] and thus a solution provides a way to understand the full dynamics. However these equations couple n-particle Green's function to $(n+1)$ - particle Green's function, thus forming an infinite heirarchy of coupled equations. Truncation procedures $[5,6]$ have to be devised to find perturbative results at some level that often lead to solutions that either ignore the transverse part of the vertex function or do not satisfy the W-T relations. Zhang [7] has pointed out that the transverse part of the vertex may play a crucial role, for example, in determining the gluon propagator by using the SchwingerDyson equation. There have been several other attempts [8-10], to define the transverse part of the vertex function based on the Lorentz structure without taking into account the symmetry of the Lagrangian. The symmetry is the fundamental constraint. Takahashi [11] suggested a way to define the transverse part of the vertex function based on symmetry, thus providing a constraint on the transverse part of the vertex.

\section{Longitudinal Ward-Takahashi Relation.}

The usual Ward-Takahashi relations [12] are well-known for the vector and the axial-vector vertex function in momentum Space. One-body W-T relation for the vector vertex function, in QED, is

$$
q_{\mu} \Gamma_{\mu \alpha}(\kappa ; \kappa+q ; q)=-i\left[S_{F}^{-1}(\kappa) \tau_{\alpha}-\tau_{\alpha} S_{F}^{-1}(\kappa=q)\right]
$$

where $S_{F}(\rho)$ is the Feynmann propagator for a fermion, $\tau_{\alpha}$ is the isosopin of the particle if it is a baryon and $\Gamma_{\mu \alpha}$ is the three point vector vertex function. For electrons, $\tau_{\alpha}=1$ and the lable $\alpha$ can be removed. This relation is a consequence of the conserved vector current (CVC) that follows as a consequence of gauge invariance and may be expressed as

$$
\partial_{\mu} J_{\mu \alpha}(x)=0
$$

or in momentum space

$$
q_{\mu} J_{\mu \alpha}(q)=0
$$

It is to be noted that in the limit $q \rightarrow 0, q_{\mu} J_{\mu \alpha}=0$.

The W-T relation may be extended to the case of partial conservation of axial current (PCAC)

$$
\partial_{\mu} J_{\mu \alpha}^{s}=\mu^{2} f_{\pi} \pi_{\alpha}(x)
$$

where $\mu$ is the pion mass, $f_{\pi}$ is the pion decay constant, $\pi_{\alpha}$ is the pion field and $J_{\mu \alpha}^{s}$ is the axialcurrent for baryon with isospin $\alpha$. PCAC leads to the W-T relation

$$
q_{\mu} \Gamma_{5 \mu \alpha}(\kappa ; \kappa q ; q)=i \mu^{2} f_{\pi} \Gamma_{\pi \beta}(\kappa, \kappa+q ; q) \Delta_{\beta \alpha}(q)
$$




$$
-i\left[S_{F}^{-1}(\kappa) \gamma_{5} \tau_{\alpha}+\tau_{\alpha} \gamma_{5} S_{F}^{-1}(\kappa+q)\right]
$$

where $\Delta_{\beta \alpha}$ is the pion propagator, $\Gamma_{5 \mu \alpha}$ is the 3-point axial-vector vertex and $\Gamma_{\pi \beta}$ is the 3-point pion vertex in momentum space. If there is an axial anamoly [13] an additional term is added to the right-hand side of Eq. (1.5)

The expression given in Eq.(1.1) and Eq. (1.5) give the consequences of CVC and PCAC respectively when there is one incoming and outging particle. For many-body systems, it is possible to extend these W-T relations for two-, three- ... and $n$-body systems. For PCAC, the weak vertex function is related to the pion vertex function. This $\mathrm{W}$-T relation has found extensive use in one- and many-body systems. [14], in particular for $\beta$-decay. These relations have been used to show explicitly cancellation of meson-exchange current effects and higher order perturbation theory results.

\section{Transverse Ward-Takahashi Relations}

Takahashi [11] suggestd a way to find constraints on the transverse part of the vertex function. Basically it is related to the idea that if the divergence and curl of a vector are known, the vector is known exactly. The Longitudinal W-T relation is a consequence of the divergence of the current which is a result of the Gauge condition. Therefore we should consider the 4-curl of the current, initially for the electromagnetic current. This idea was pursued by He and Khanna [15]. In this paper the transverse W-T relation was derived in coordinate space and then a Fourier transform brought a neater form.

$$
\begin{array}{r}
i q^{\mu} \Gamma_{v}^{v}\left(p_{1}, p_{2}\right)-i q^{v} \Gamma_{v}^{\mu}\left(p_{1}, p_{2}\right) \\
=S_{F}^{-1}\left(p_{1}\right) \sigma^{\mu v}+\sigma^{\mu v} S_{F}^{-1}\left(p_{2}\right)+2 m \Gamma_{T}^{\mu v}\left(p_{1} \cdot p_{2}\right)+\left(p_{1 \lambda}+p_{2 \lambda}\right) \varepsilon^{\lambda \mu \nu \rho} \Gamma_{A \rho}\left(p_{1}, p_{2}\right)
\end{array}
$$

where $q=p_{1}-p_{2}, \Gamma_{v}^{\mu}, \Gamma_{A}^{\mu}$ and $\Gamma_{T}^{\mu v}$ are the 3-point vector, axial-vector and tensor vertex functions, respectively. This relation was shown to work at three tree level. However this relation failed at one-loop level due to the neglect of a higher order contribution that is essential at loop level.

This deficiency was corrected in a recent paper [16]. It is important to emphasize that the curl of the current is being calculated i.e.

$$
\partial_{x}^{\mu}<0\left|T\left(j^{v}(x) \Psi\left(x_{1}\right) \bar{\Psi}\left(x_{2}\right)\right)\right| 0>-\partial_{x}^{v}<0\left|T\left(j^{\mu}(x) \Psi\left(x_{1}\right) \bar{\Psi}\left(x_{2}\right)\right)\right| 0>
$$

A term involving Wilson line $U_{\rho}(x \prime, x)=P \exp \left[-i g \int_{x}^{x \prime} d y^{\rho} A_{\rho}(y)\right]$ was left out. This adds the following term in momentum space to the expression in Eq.(2.1).

$$
-\int \frac{d^{4} k}{(2 \pi)^{4}} 2 \kappa_{\lambda} \varepsilon^{\lambda \mu v \rho} \Gamma_{A \rho}\left(p_{1}, p_{2} ; \kappa\right)
$$

where $\kappa$ is the internal momentumof the gauge boson appearing in the Wilson line. This leads to higher order corrections to $\Gamma_{A \rho}\left(p_{1}, p_{2}\right)$. This expresion has been tested to one-loop order. The results agree with the perturbative calculations carried out earlier. The exact result has not been tested beyond one loop level but it will work to all orders. 
Therefore we have now both the longitudinal and transverse part of the vertex and both are in a non-perturbative form and should be satisfied at any order of perturbation theory. It is to be emphasized that the transverse part not only satisfies the Lorentz structure but also the overall symmetry of the theory manifest in the Schwinger-Dyson equation. For further details the reader is referred to the original papers $[15,16]$.

\section{Full fermion-gauge-boson vertex}

To get the complete fermion-gauge-boson vertex, we put together the expression in Eq. (1.1) with Eq. (2.1) and Eq. (2.2) to get the result. Multiply Eq. (2.1) and Eq. (2.2) by $i q_{v}$ to get

$$
\begin{aligned}
q_{v} q^{v} \Gamma_{v}^{\mu}\left(p_{1}, p_{2}\right)-q^{\mu} q_{v} \Gamma_{v}^{v}\left(p_{1}, p_{2}\right) & =i q_{v} S_{F}^{-1} \sigma^{\mu v}+i q_{v} \sigma^{\mu v} S_{F}^{-1} \\
& +2 m i q_{v} \Gamma_{T}^{\mu v}\left(p_{1}, p_{2}\right)+i q_{v}\left(p_{1 \lambda}+i q_{v}\left(p_{1 \lambda}+p_{2 \lambda}\right) \varepsilon^{\lambda \mu \nu \rho} \Gamma_{A \rho}\left(p_{1}, p_{2}\right)\right. \\
& -i q_{v} \int \frac{d^{4} \kappa}{(2 \pi)^{4}} 2 \kappa_{\lambda} \varepsilon^{\lambda \mu v \rho} \Gamma_{A \rho}\left(p_{1}, p_{2} ; \kappa\right)
\end{aligned}
$$

Now use Eq. (1.1) for $q_{v} \Gamma_{v}^{v}$, to get

$$
\begin{aligned}
\Gamma_{v}^{\mu}\left(p_{1}, p_{2}\right) & =\frac{1}{q^{2}}\left[-i q^{\mu}\left(S_{F}^{-1}\left(p_{1}\right)-S_{F}^{-1}\left(p_{2}\right)\right)+i q_{v} S_{F}^{-1} \sigma^{\mu v}+i q_{v} \sigma^{\mu v} S_{F}^{-1}\right. \\
& +2 m i q_{v} \Gamma_{T}^{\mu v}+i q_{v}\left(p_{1 \lambda}+p_{2 \lambda}\right) \varepsilon^{\lambda \mu v \rho} \Gamma_{A \rho} \\
& \left.-i q_{v} \int \frac{d^{4} \kappa}{(2 \pi)^{4}} 2 \kappa_{\lambda} \varepsilon^{\lambda \mu v \rho} \Gamma_{A \rho}\right]
\end{aligned}
$$

This gives the full fermion-gauge-boson vector vertex. Similarly transverse components of the axial-vector, tensor and pseudo scalar vertex can be obtained. Then the full vertex can be written down.

It is to be noted that in the chiral limit i.e. $m=0$, only the vector and the axial-vector vertices are coupled and thus these have to be calculated together in the non-chiral limit i.e. $m \neq 0$, all the vertex funcions are calculated simultaneously.

\section{Conclusion}

We have derived the transverse vector fermion-gauge-boson vertex that allows us to write a complete vector vertex. The general use of these relations is stil not established.

The extension of these results to non-abelian gauge theories still needs to be considered. The fact that such a quantum field theory cannot be treated in a canonical approach may provide some difficulties. One has to work in the path integral approach. The corresponding W-T relations for non-abelian theories are much more difficult to obtain and are rather cumbersome to use.

It is important to note that $\mathrm{W}-\mathrm{T}$ relations have been useful in finite temperature quantum field thories. They have played an important role in defining the Goldstone bosons arising from Spontaneous Symmetry breaking. It will be interesting to cast the results given above for the finite temperature theories. 
Finally it is rather interesting to note that symmetry of the Lagrangian and spontaneous symmetry breaking have played an interesting role in our understanding of phenomenon in physics. The earliest use of spontaneous symmetry breaking may be viewed for phenomenon of superconductivity and spin waves in condensed matter physics. The pressure of symmetry which leads to the Ward-Takahashi relations provide non-perturbative results that are really independent of any detailed structure of the interactions. These must be sorted out first before resorting to any sort of perturbation scheme that, by necessity, will depend on the nature and strength of the interaction. We have demonstrated, in detail, the role of symmetry in establishing a complete understanding of the vertex for gauge fields and for partially conserved axial vector current. These results find extensive use in many-body physics, in particular systems with strong interactions.

The author wishes to thank the organisers of the Fifth International Conference on Mathematical Methods in Physics in Rio de Janeiro for giving me the opportunity to present these ideas. I thank Prof. H.X. He for a pleasant collaboration and help. The research is supported by Natural Sciences and Engineering Research Council of Canada.

\section{References}

[1] J. Ward, Phys. Rev. 78 (1950) 182; Y. Takahashi, Nuovo Cimento 6 (1957) 370.

[2] J. Goldstone, A. Salam, S. Weinberg, Phys. Rev. 127 (1962) 965.

[3] H.X. He, F.C. Khanna, H. Umezawa, Ann. Phys. (NY) 256 (1997) 144.

[4] F.J. Dyson, Phys. Rev. 75 (1949) 1736; J. Schwinger, Proc. Nat. Acad. Sci. 37 (1951) 452; 455.

[5] A. Cohon, H. Georgi, Nucl. Phys. B314 (1989) 7; W. Bardeen, C.N. Leung, S.T. Love, Nucl. Phys. B323 (1989) 493.

[6] A. Salam, Phys. Rev. 130 (1963) 1287; R. Delbourgo, Nuovo Cimento 49A (1979)484.

[7] Z.R.B. Zhang, Phys. Rev. D31 (1985) 1512.

[8] J.S. Ball, T.W. Chiu, Phys. Rev. D22 (1980) 2542.

[9] J.E. King, Phys. Rev. D27 (1983) 1821.

[10] D.C. Curtis, M.R. Pinnington, Phys. Rev. D42 (1990) 4165.

[11] Y. Takahashi, Phys. Rev. D15 (1977) 1589; in Quantum Field Theory; F. Mancini (Ed.) Elsevier Publisher, 1988; P19.

[12] J.C. Ward, Phys. Rev. 78 (1950) 182; Y. Takahashi, Nuovo Cimento 6 (1957) 371.

[13] S.L. Adler, Phys. Rev. 177 (1969) 2426; J.S. Bell, R. Jackiwe, Nuovo Cimento 60A (1969) 47.

[14] X.Q. Zhu, S.S.M. Wong, F.C. Khanna, Y. Takahashi, T. Toyoda, Phys. Rev. C36 (1987) 1968.

[15] H.X. He, F.C. Khanna, Y. Takahashi, Phys. Lett. B480 (2000) 222; H.X. He, Phys. Rev. C63 (2001) 025007.

[16] H.X. He, F.C. Khanna, Int. J. Mod. Phys. 21 (2006) 2541. 